\title{
RHIC VACUUM INSTRUMENTATION AND CONTROL SYSTEM ${ }^{*}$ R.C. Lee, D. Pate, L. A. Smart ${ }^{\dagger}$ D. Weiss, D. Zigrosser, RHIC Project, BNL, Upton, NY
}

\section{Abstract}

The Relativistic Heavy Ion Collider (RHIC) Project is a nuclear physics research accelerator at Brookhaven National Laboratory (BNL) with beam circulation scheduled for May 1999. To achieve the necessary beam lifetime, the vacuum in the two concentric rings must be constantly monitored to isolate problem vacuum regions. One function of the RHIC vacuum instrumentation and control (I\&C) system is to operate sector valves with inputs from several hundred gauges and pumps distributed around the $3.8 \mathrm{~km}$ circumference of the accelerator rings. The architecture and implementation of the RHIC vacuum I\&C system from the pumps and gauges to the remote user interfaces are described.

\section{RHIC VACUUM SYSTEMS}

There are three distinct vacuum systems in RHIC proper (see Fig. 1). The insulating cryostat vacuum houses the helium-cooled super-conducting magnets. The cold beam tube vacuum passes through the center of the magnets. The warm beam tube vacuum, which connects magnet strings, makes up roughly $16 \%$ of the RHIC beam line.

Ions from the linear accelerator or Tandem Van de Graaff are accelerated by the Alternating Gradient Synchrotron/Booster, and injected into one of the two RHIC rings. RHIC has six equidistant sites for beam collision detection.

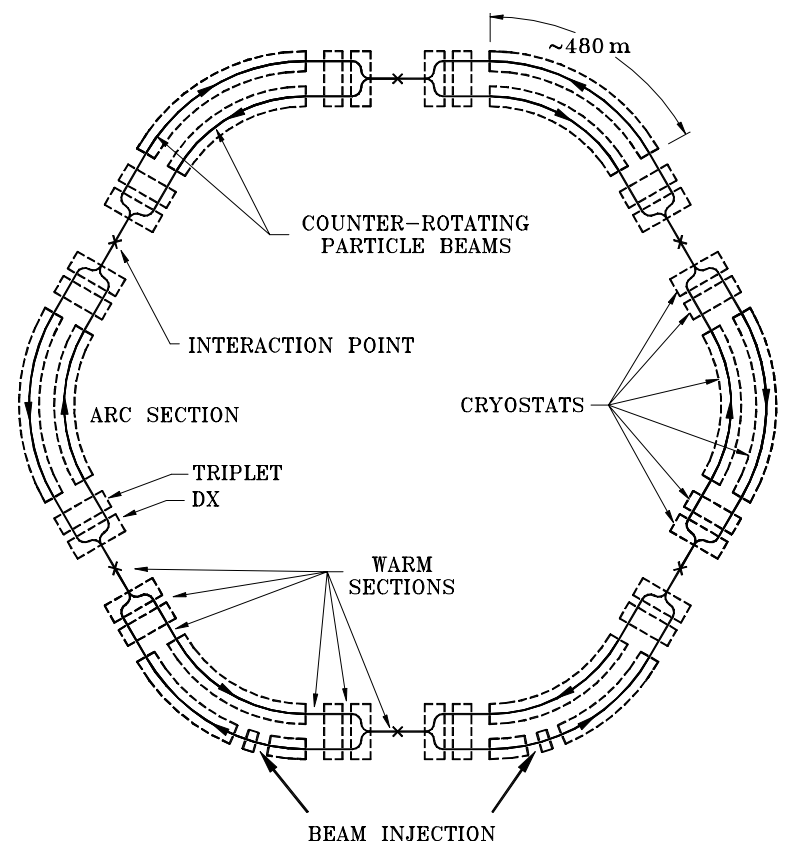

Figure 1: The RHIC layout

\footnotetext{
* Work performed under the auspices of the U.S. Energy Department.

${ }^{\dagger}$ Email: 1smart@bnl.gov
}

\subsection{Insulating Cryostat Vacuum System}

The insulating vacuum must be kept below $10^{-5}$ Torr to minimize heat transfer to the $4.2 \mathrm{~K}$ superconducting magnet cold mass. From earlier test measurements, the cryostat total pressure falls into the low $10^{-7}$ Torr range[1]. The cold mass of the superconducting magnets pumps all but the lightest gases, so turbomolecular pumps (TMPs) with mechanical backing pumps are employed. Inverted magnetron cold cathode gauges (CCGs) and convectionenhanced Pirani gauges are used to monitor the cryostat pressure. Partial pressure analyzers (PPAs) are used to sample the gas composition of the cryostat and beam vacuum environments and can give an indication of helium leaks from magnet vessels or air leaks in the cryostat after cool-down.

\subsection{Warm Bore Vacuum System}

For the ten-hour-plus beam lifetime, the average ambient (or warm) beam vacuum must be below $5 \times 10^{-10}$ Torr. A warm sector is the warm bore between two sector valves. Sector valves are all-metal gate valves used to isolate the warm beam tube vacuum from adjacent warm sectors or from the cold beam tube. Ion pumps are installed in the warm beam vacuum sectors. Each ion pump has two pockets into which titanium sublimation pump (TSP) cartridges are installed. There are fifty-two distinct warm sectors in RHIC proper. The number of ion pumps in each sector depends on the gas load of various chambers. Prior to installation, warm bore gauges are baked and leak checked on their instrument trees.

\subsection{Cold Bore Vacuum System}

The average cold beam vacuum must be less than $1 \times 10^{-11}$ Torr. The cold beam tube or cold bore, is passively pumped by the cold tube walls and charcoal-filled sorption pumps that are installed at $\sim 30 \mathrm{~m}$ intervals. The cold bore temperature is $\sim 4.5 \mathrm{~K}$ and the sorption pump temperature is less than $10 \mathrm{~K}$. The pressure in the cold bore is monitored by gauges that are also $\sim 30 \mathrm{~m}$ apart. Small americium alpha sources are installed inside the cold bore CCG tubes to help start the gauge discharge at very low pressures [2]. This modification allows a single type of gauge to be used for all of the ring vacuum systems. 


\section{THE VACUUM I\&C SYSTEM}

The heart of the vacuum I\&C system is a peer-to-peer network of programmable logic controllers (PLCs). The control of vacuum devices is divided geographically among eight PLCs. The PLCs take care of the safe operation of the vacuum system, and are the link between the intelligent gauge and pump controllers and the controls system front-end computers (FECs). Data from the FECs is transmitted to remote Console-Level Computers (CLCs) where applications are available to display and log vacuum system parameters. A bottom-up description of the system follows.

\subsection{The Instrumentation}

There are over one thousand gauges and ion pumps in the RHIC vacuum systems. Although all interface with the controls system, only two thirds control $\sim 125$ sector valves. Controllers for gauge and ion pumps whose pressures directly affect the operation of valves are located in one of eight service buildings in racks adjacent to the vacuum PLC. Cables are run from gauges and pumps in the RHIC tunnel to the service building several hundred feet away (see Fig 2). A normally open set point relay is associated with each gauge. When the gauge pressure is below the selected set point level, the relay contact is closed indicating good vacuum. The rest of the gauge controllers are installed in the accelerator tunnel; one is incorporated in each TMP station. The PPAs have the electronic control unit directly mounted on the sensor. All TMPs and PPAs are located in the tunnel.

\subsection{The PLC}

Each PLC chassis comprises one processor module, one or two communication coprocessors with serial expander modules, and several input and output (I/O) modules.

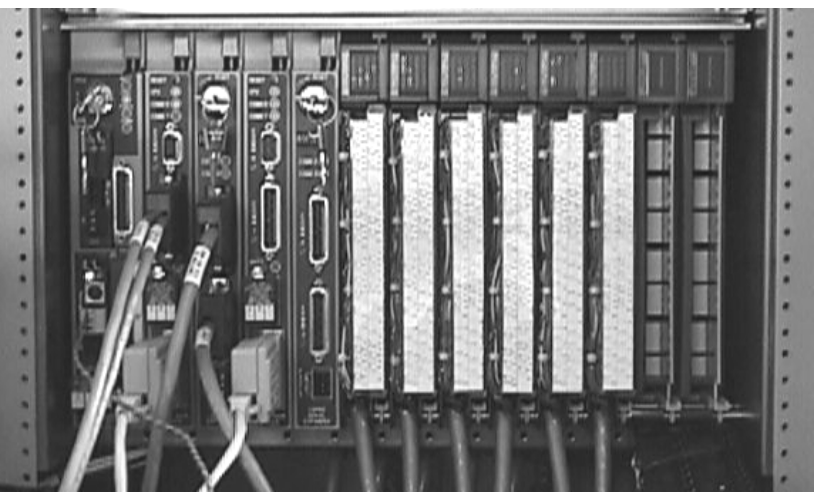

Figure 2: A typical PLC chassis

Data is transferred between PLCs on a high-speed Data Highway Plus ${ }^{\mathrm{TM}}$ serial communication networks [3]. Each coprocessor with serial expander has six serial communication ports assigned to five types of vacuum devices: gauge controllers, ion pump controllers, TMPs, PPAs, and TSPs. The I/O modules have either 32 inputs or outputs, with control voltages ranging from 10 to $30 \mathrm{Vdc}$.

Ladder logic programs reside in the PLC processor module. The ladder programs control the valve solenoids based on user inputs and the input module signals from gauges, pumps, and valves. Each sector valve has open and closed limit switches wired to the PLC inputs and a solenoid that is wired to the PLC output module. A valve can be opened only if the vacuum level satisfies gauge set points on both sides of the valve. Valves are closed by the PLC under a number of conditions, such as when the vacuum is bad or multiple controllers fail.

\subsection{The Beam Permit Link}

Beam can only be introduced into a RHIC ring if accelerator system safety precautions are met. One of these measures, the vacuum system beam permit link (BPL), provides a positive indication that all sector valves for a

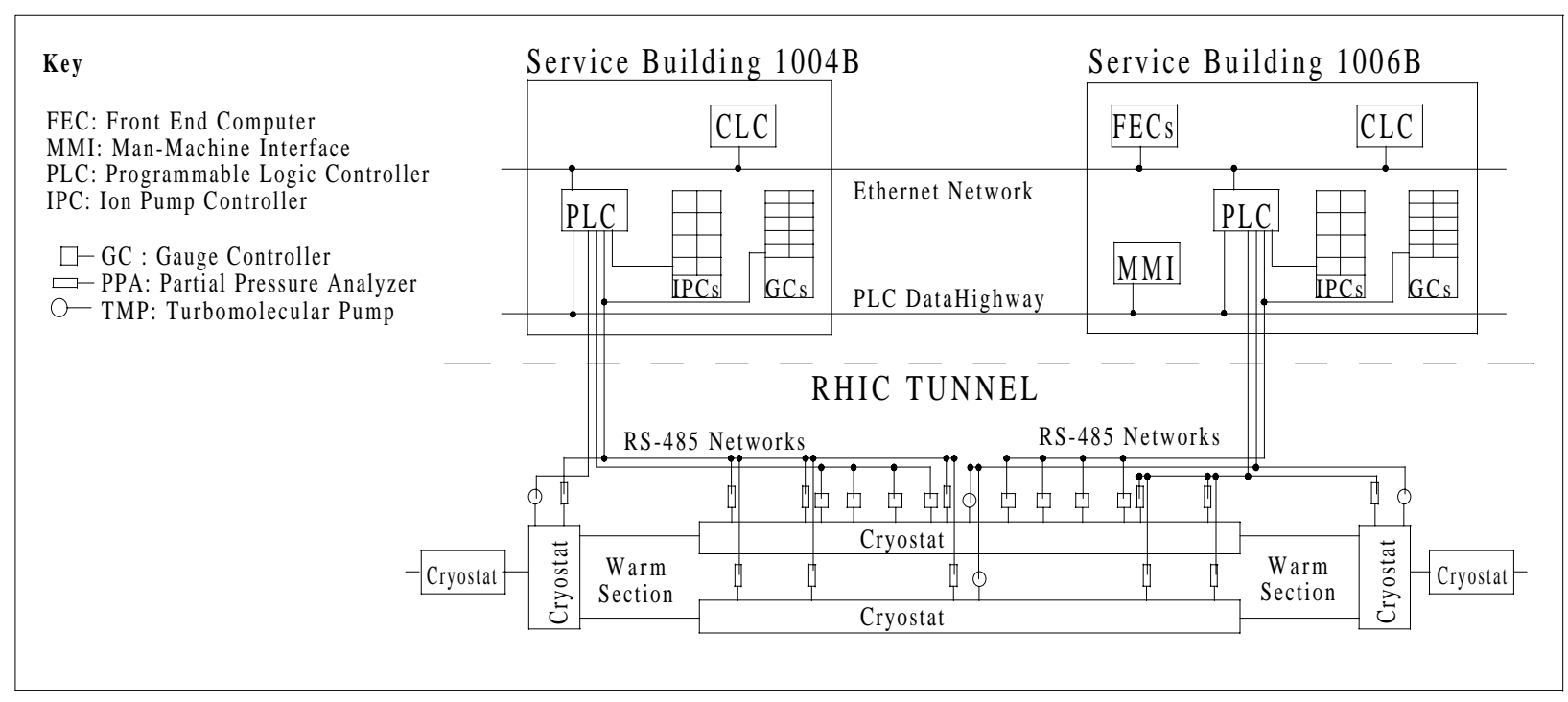

Figure 3: System architecture for one sixth of RHIC. 
given ring are open. There are permit signals for both rings at each vacuum system PLC. If a single valve closes, or the limit switches do not indicate that the valve is open, then the beam permit is removed. The BPL will also inhibit beam if there is a failure of the PLC or its power source.

Two of three pressure readings must rise above the set points to close a sector valve, causing the PLC to remove the BPL. A single high pressure reading will not trip the BPL. To minimize the chance of removing the beam permit during short power outages, the PLC and associated power supplies are backed by an uninterruptible power supply.

\subsection{Remote Monitoring and Control}

In addition to protecting the ring from injecting beam with valves closed, the I\&C system must provide remote control of devices.

C-language programs in the PLC coprocessor poll gauge controllers, ion pump controllers, TMP stations and PPAs on five networks (the gauge controllers are divided on two networks due to the number of devices). Distinct networks are needed because each device has a unique command structure or protocol. For example, the gauge controllers and TMP stations reply in ASCII characters, the ion pumps talk back in hexadecimal codes, and the PPAs use a 9-bit protocol that manipulates the parity bit. The coprocessor software receives the data in many formats and re-packages it for the controls system FECs.

The coprocessor has an Ethernet port through which data is uploaded to the control section FEC. Since all data transfer between the FEC and the vacuum system occurs through the coprocessor, the data in the PLC processor memory must be copied to coprocessor memory for FEC retrieval. Similarly, commands from the FEC are routed through the coprocessor to the PLC.

\subsection{The Front-End Computer}

There are four FECs for the RHIC vacuum devices. The FECs are VME based processors with up to 32 Mbytes of memory. All data from the vacuum system to the Controls System, including PLC I/O status, is routed through the PLC coprocessor Ethernet port. That data is exchanged at a rate of $10 \mathrm{Mb} / \mathrm{s}$.

\subsection{The Console-Level Computer}

The CLCs are UNIX workstations that run applications to display and log the FEC. One application, Parameter Editing Tool, is a general-purpose program developed at BNL for viewing and modifying RHIC control system parameters. Users can see gauge pressures, ion pump current, and the status of valves or TMP stations in spreadsheet format. The user can also send commands to manipulate sector valves, change PPA parameters, turn ion pumps on/off, or control TMP stations valves and pumps. Other applications perform data logging and archiving tasks. The CLC and FEC exchange data at a rate of $100 \mathrm{Mb} / \mathrm{s}$ over the Ethernet network.

\subsection{The Man-Machine Interface}

A back-door provision for the control and monitoring of the vacuum devices was built into the system in the event that the FEC or Ethernet link fails. There is another user interface called the MMI (man-machine interface) that is independent of the controls system computers. The MMI is an industrial computer with software that interfaces directly with all PLCs on the DataHighway network. This allows control all RHIC vacuum sector valves from a limited number of locations (presently two) should the FECs or CLCs become unavailable. All gauge relay contact and valve limit switch data is displayed in a graphic format, so that the state of the vacuum system can be determined at a glance. The MMI currently does not have the capability to display remote serial data, but it does provide an alternate means of determining the status of the entire vacuum system's readiness for beam.

\section{CURRENT STATUS}

To date, over $85 \%$ of gauge controllers, ion pump controllers, and TMPs are reporting device readings to the controls system. Pressure readings from $250+$ gauge controllers throughout the vacuum system are being monitored. Over ninety percent of the ion pumps are operational with most warm sectors at high vacuum. PPAs are also on-line, reporting partial pressure readings in a few warm regions. All cold bore beam tubes are under rough vacuum in preparation for the cool-down of the magnet strings. All PLC processors and co-processors are programmed and operational. Controls system data logging and plotting software is also operational. The BPL signals from the PLCs are wired to the controls system inputs in preparation for accelerated beam in May.

\section{AKCNOWLEDGEMENTS}

The authors would like to credit Robert Burns for the design of the vacuum instrumentation and control system architecture. We also thank the Vacuum System Section staff for the years of effort that went into assembling and installing the system, and for making it work.

\section{REFERENCES}

[1] H.C Hseuh, et al., Proc. 14 ${ }^{\text {th }}$ Int'l Vac. Congress, Birmingham UK, Sept. 1998.

[2] K.M.Welch, et al, J. Vac. Sci. Technol A 14(3), May/Jun 1996.

[3] DataHighway is a trademark of Rockwell Automation (Allen-Bradley brand). 